\title{
Prescribing Pattern of Ceftriaxone among Inpatients Admitted to Emergency Room in Military Hospital-Omdurman, Sudan
}

\author{
Hussien Faki LA ${ }^{1}$ and Awad MM2* \\ ${ }^{1}$ Omdurman Islamic University, Sudan \\ ${ }^{2}$ Assistant professor of Pharmacy Practice at International University of Africa (IUA), \\ Sudan
}

*Corresponding author: Mohamed Mousnad, Consultant of Pharmacoeconomics \&

Pharmacoepidemiology, assistant professor, Faculty of Pharmacy, International University of Africa (IUA), Khartoum, Sudan, Tel: +249-912325864; Email: m_abdalaziz@yahoo.com

\section{Abstract}

Objectives: A new onset of ceftriaxone resistance is serious complication of therapy which is leading to a new treatment strategies or drugs. The aim of this study was to identify ceftriaxone regimens, estimate the cost of it per prescription and determine the prescriber adherence to ceftriaxone prescription protocol.

Methods: A retrospective observational hospital-based study, conducted in emergency room at Military HospitalOmdurman in period from October 2017 to March 2018, on patients who took ceftriaxone. The data collected by using a pretested designed data collection sheet. The collected data were analyzed by SPSS version (23).

Results: A total of 90 patients were included, out of them $93.30 \%$ of patients received ceftriaxone for other conditions rather than for meningitis, pneumonia and enteric fever. The majority of studied patients were in the age group more than 60 years (35.6\%), (50\%) were females. Almost all patients studied did not make culture and sensitivity test and prescribed by brand name. Most patients were dosed as $1 \mathrm{~g}(83.30 \%)$ and duration of therapy was found to be high in the range 5-7 days and 7-14 days (32.2\%). Most of the interaction made by co-administration of other drugs with ceftriaxone was serious (50\%).

Conclusions: The cost was found to be high. The prescribing pattern of ceftriaxone according to it is regimens written by doctors, the cost and adherence of the prescriber to ceftriaxone prescription protocol reveal that irrational use of ceftriaxone or improper use.

Keywords: Antibiotics; Pneumonia; Anti-Microbial Policy

\section{Introduction}

The cephalosporins are the largest and most diverse family of antibiotics of the beta-lactam group. It grouped into "generations" based on their spectrum of antimicrobial activity [1]. Third-generation cephalosporin with broad-spectrum gram-negative activity; has lower efficacy against gram-positive organisms but higher efficacy against resistant organisms. Ceftriaxone is a broad-spectrum third generation cephalosporin antibiotic 


\section{Journal of Quality in Health Care \& Economics}

and it is one of the most commonly used antibiotics due to its high antibacterial potency, wide spectrum of activity and low potential for toxicity [2].

Because of the broad spectrum use of antibiotic especially the over use of cephalosporins and also the misuse of them led to antimicrobial resistance. Therefore, prudent prescribing of antimicrobial drugs is essential as it may reduce incidences of antimicrobial drug resistance [3]. Recent studies revealed resistance of E.coli and Klepsilla spp. To ceftriaxone in Khartoum teaching and Soba university hospital ranged from $(56.5 \%$ - 79\%) respectively. Close monitoring of the prescribing pattern of ceftriaxone is very important to preserve it is susceptibility.

WHO reports widespread resistance to older and cheaper antibiotics. The development of new antibiotics is not very attractive for commercial pharmaceutical companies. Treatments are taken only for short periods of time (unlike medicines for chronic disease) and they become less effective as resistance develops, meaning that the supply of new drugs constantly needs to be replenished.

The state ministry of health in the state of Khartoum was stated antimicrobial policy that prepared by the anti microbial policy committee for controlling the prescribing of antimicrobial agents and this license is categorized into four different levels namely unrestricted antimicrobials, restricted, reserved antimicrobials and special situations. Antimicrobials known to cause collateral damage must be restricted to special condition only. Ceftriaxone is a sample of that and must be approved by the head of the unit only for meningitis, pneumonia and enteric fever. The clinician must record the antimicrobial dose, route of administration, frequency and duration of treatment in the patient's note and the treatment sheet [4].

\section{Literature Review}

Statistics gathered by researchers showed that resistance to ceftriaxone was largely developed within some of bacteria that ceftriaxone kill. In addition overuse, misuse and use of ceftriaxone without culture to test the susceptibility will finally lead to loss the effectiveness and thereby wastage of resources, account for a large portion of a hospital's pharmacy budget, augment risk for adverse drug reaction and additional economic burden on patients [5-7].

There is no research done in Khartoum about this issue to reflect the current situation. The proposed current study may evaluate the utilization of ceftriaxone and would like to fill this gap.

Many studies worldwide were done to determine the prevalence and antimicrobials susceptibility of multi-drug resistance (MDR) Escherichia coli (E.coli) collected from clinical specimens of patients in different hospitals in Khartoum State. It also investigated the possible differences in antimicrobial resistance of E.coli isolated from a various type of clinical specimens in relation to the patient sex, age and settings. Prospective study was done at Sudan and limited data are currently available. The study was conducted on 232 various clinical specimens collected from different hospitals in Khartoum State. High resistance rates were observed to amoxicillin, cefuroxime, trimethoprim-sulfamethoxazole, tetracycline, nalidixic acid and ceftriaxone (64\%). Isolates from males were showed higher resistance rates than those from females to ceftriaxone, but according to age showed no statistical differences between adults and children. They conclude that Escerichia coli (E.coli) is a common pathogen linked with community associated as well as nosocomial infections. In the last few years, the emergence and wide dissemination of E.coli strains showing resistance to broad-spectrum of antimicrobial agents has been reported [8-10].

\section{Materials and Methods}

This study was conducted in emergency room (ER) at Military Hospital-Omdurman in Khartoum state (Sudan) between October 2017 and March 2018; because there is no the Hospital does not have a system that shows how many patients are taking ceftriaxone. Data collection through pretested designed data collection sheet. Patients analyzed in this study were Sudanese patients registered in Omdurman Military Hospital databases as at emergency room patients on ceftriaxone with or without insurance coverage were selected. Demographic, clinical and laboratory data was recorded from the patients' medical charts and files.

All available medical charts and files of patients who took ceftriaxone and admitted to ER between October 2017 and March 2018 at Military Hospital-Omdurman. 90 patients were included in the analysis. Data was entered and analyzed by using the statistical package for social science (SPSS) version [11]. Tests were done using a priori level of significance 0.05 . All results are expressed in U.S. dollar and the central bank of Sudan exchange rate at the time of the study was 1 USD $=45$ SDG (19.07.2018). 


\section{Results}

\section{Indications of Prescribing Ceftriaxone}

Figure 1 displays the most common indication of ceftriaxone. It was others such as septicemia, Urinary Tract Infection and gastrointestinal disease $(93.30 \%)$ followed by pneumonia (3.30\%), meningitis $(2.20 \%)$ and enteric fever $(1.10 \%)$.

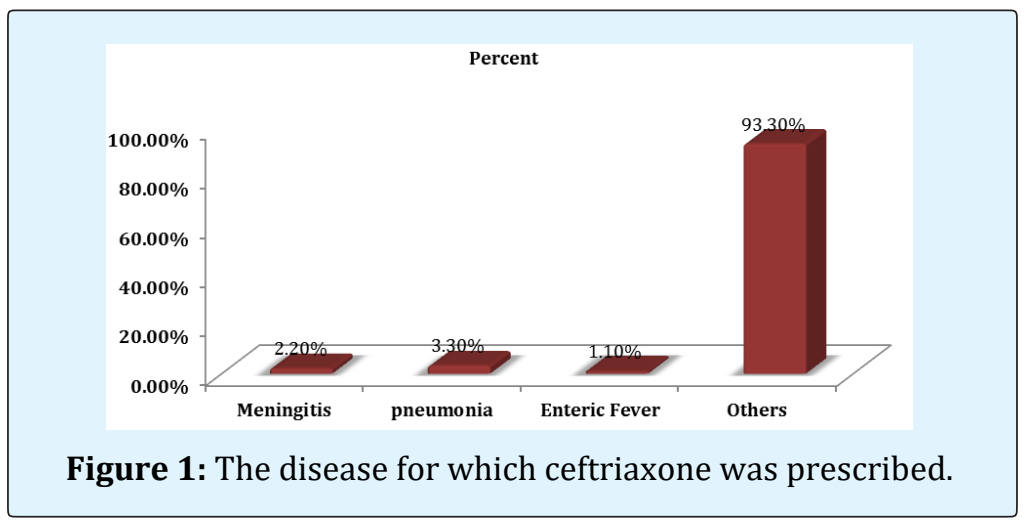

\section{Culture and Sensitivity Test of Ceftriaxone}

Figure 2 shows ceftriaxone was often used without making the culture and sensitivity test in emergency room. In a study population of 90 patients from emergency room 88 (97.8\%) patients were not making culture and sensitivity test; so that just 2 patients were making culture and sensitivity test (2.20\%).

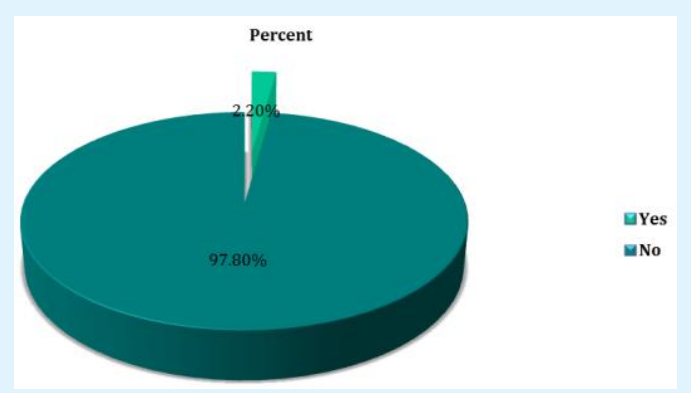

Figure 2: Culture and sensitivity test of ceftriaxone among studied patients.

\section{Ceftriaxone Doses}

Figure 3 displays mostly, ceftriaxone was given $1 \mathrm{~g}$ which covered $(83.30 \%)$, followed by $2 \mathrm{~g}$ that covered
(11.10\%), 500mg (3.30\%) and 250mg and other doses $(1.10 \%)$ was prescribed.

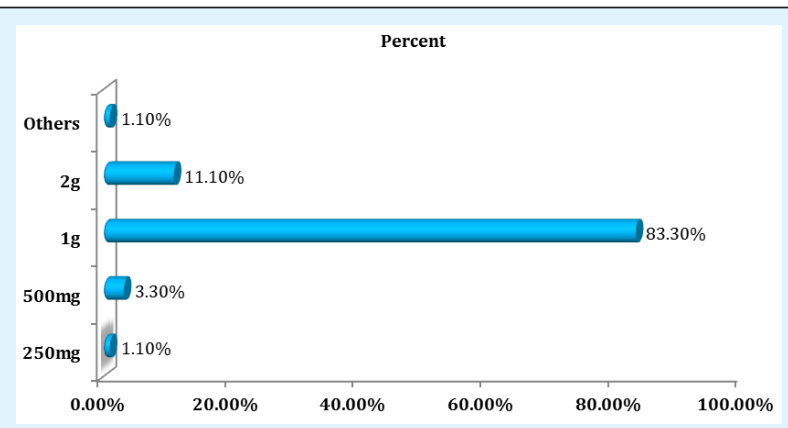

Figure 3: Ceftriaxone dosing distribution of patients included in the study. 


\section{Duration of Ceftriaxone Therapy}

Figure 4 represents of all studied patients the vast majority had taken ceftriaxone for a period of 5-7 days and $7-14$ days $(32.2 \%)$.

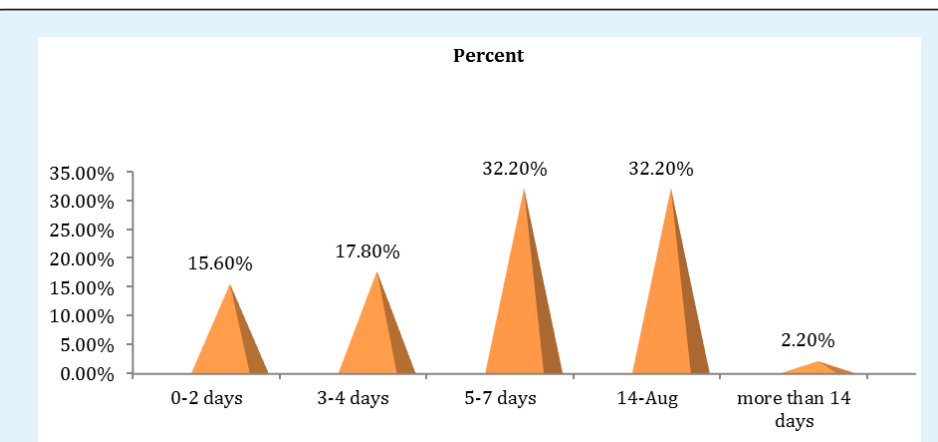

Figure 4: Duration of ceftriaxone therapy distribution.

\section{Clear Regimen of Ceftriaxone from Initial Plan}

$98.9 \%$ of patients did not have a clear regimen of ceftriaxone from the beginning of treatment.

\section{Co-Administration with Ceftriaxone}

Figure 5 displays among the prescription, other drugs were commonly co-administered (95.6\%) with ceftriaxone rather than just ceftriaxone in the prescription (4.40\%).

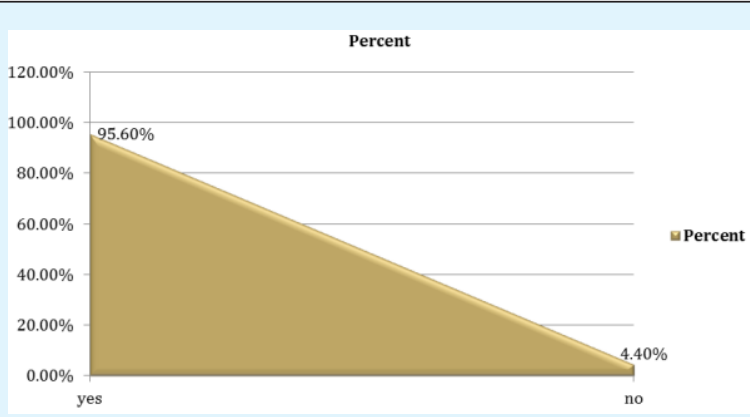

Figure 5: Availability of co-administration of other drugs with ceftriaxone.

\section{Co-Administered Drugs's Interaction with Ceftriaxone}

Table 1 shows concerning interaction of ceftriaxone with co-administered drugs, (33.3\%) of regimen had interaction with ceftriaxone, and almost of patients were serious interaction $(50 \%)$.

\begin{tabular}{|c|c|c|}
\hline & Frequency & Percent \\
\hline Yes & 30 & $33.30 \%$ \\
\hline No If yes, what is the severity of the interaction ( n =30) \\
\hline \multicolumn{2}{|c|}{$66.70 \%$} \\
\hline Contraindicated & 6 & $20 \%$ \\
\hline Serious & 15 & $50 \%$ \\
\hline Monitor closely & 2 & $6.70 \%$ \\
\hline Minor & 7 & $23.30 \%$ \\
\hline Total & $\mathbf{3 0}$ & $\mathbf{1 0 0}$ \\
\hline
\end{tabular}

Table 1: Distribution of the types of interaction that happened when co-administered other drugs with ceftriaxone. 


\section{Journal of Quality in Health Care \& Economics}

\section{The Patient Insurance and the Cost Afforded by Ceftriaxone Per Prescription}

Table 2 displays that $(87.8 \%)$ of patients had military insurance and just (12.2\%) were without this insurance.
The highest cost afforded for ceftriaxone per patient in emergency room was found to be more than 2 U.S. dollar $(90.9 \%)$ and (9.1\%) cost between 1-2 U.S. dollar.

\begin{tabular}{|c|c|c|}
\hline & Frequency & Percent \\
\hline Yes & 79 & $87.80 \%$ \\
\hline No & 11 & $12.20 \%$ \\
\hline If no, how much does it cost per day per U.S. dollar ( n = 11) \\
\hline 1 - 2 USD & 1 & $9.10 \%$ \\
\hline more than 2 USD & 10 & $90.90 \%$ \\
\hline Total & $\mathbf{1 1}$ & $\mathbf{1 0 0}$ \\
\hline
\end{tabular}

Table 2: The patients with insurance or not and the variation of cost afforded for ceftriaxone per patient among studied cases.

\section{Discussion}

The irrational use of ceftriaxone was $(93.30 \%)$ rather than treatment of main indications of use of ceftriaxone, followed by pneumonia (3.30\%), meningitis $(2.20 \%)$ and enteric fever $(1.10 \%)$. This pattern was similar in trend obtained in North East Ethiopia. This can be attributed to the fact that doctors gave the patients ceftriaxone according to their experience. In many cases ceftriaxone was prescribed for prophylactic rather than for treatment and ceftriaxone abuse such as this in the community and hospital fuels the crisis of ceftriaxone resistance.

Culture and sensitivity test was not done in most of the patients (97.8\%). This in line with Tikur Anbessa Specialized Hospital where they reported the culture and sensitivity test was $(89.5 \%)$. The interviewed physicians agreed that culture and sensitivity tests were not done in majority of cases and this was ascribed by unavailability of service, unreliable culture result, prior initiation of ceftriaxone regimen and delayed culture result. The interviewed microbiologists agreed that the reason the microbiology results were unconvincing could be due to sample collection, improper use of transporting medium and failure to request appropriate laboratory test. They agreed that the quality of the current microbiology laboratory is poor due to mainly poor quality reagents and it takes on average, 3 days for culture results to become available.

The doses most prescribed were $1 \mathrm{~g}(83.30 \%)$ and $2 \mathrm{~g}$ $(11.10 \%)$. The most common duration of treatment was 5-7 days and 7-14 days (32.2\%). The results obtained differ slightly from that done in Ghana, this difference might come back to the fact that the study conducted in Ghana was consider patient outcome and the evaluation of patients drug therapy before medication was dispensed which can be mentioned as one of the drawback of this study. Also may be according to the difference in patient condition; patients who admit to the hospital may be those who are in most cases terminally-ill requiring longer hospital stay and this in turn may cause physicians to give ceftriaxone to the patients for long duration of treatment. Also doctors were written the dose and frequency of ceftriaxone daily in patient's file, not from the beginning of treatment.

The major results collected conclude that the dose, frequency and duration of ceftriaxone were not well defined at the initial plan (98.9\%), which may lead to that this study was conducted in a small population, for a small duration of time. This would not take into consideration any seasonal variations and carried out only in emergency room.

About $95.6 \%$ of ceftriaxone was co-administered with other drugs (for example antibiotics and supplements). But $66.7 \%$ which showed interaction recorded. Among the medication co-administered with ceftriaxone, serious interaction took first place $(50 \%)$ followed by minor interaction $(23.3 \%)$, contraindication with ceftriaxone (20\%) and monitor closely (6.7\%) as clarified in study done in Addis Ababa. This may indicate the prevalence of disease and co-morbidities in which ceftriaxone was coadministered with other drugs. And this in turn indicates the presence of medication related problem, namely IV incompatibility with respect to ceftriaxone use. Additionally the interviewed physicians agreed that such practice was due to the less availability of other drugs and absence of checking for possible interaction before prescribing. Because interactions with ceftriaxone occur 


\section{Journal of Quality in Health Care \& Economics}

lead to drug resistance and inefficiency and become unhelpful.

Ceftriaxone was account for $87.8 \%$ of the medical insurance budget in Omdurman Military Hospital. 90.9\% of patients did not have medical insurance pay more than USD 2 per day for the hospital to get ceftriaxone. Pressure to reduce the cost of antimicrobial therapy is especially intense because these drugs may account for a large portion of hospital's pharmacy budget and patient budget. This findings focus on the reality of in-appropriate use in the treatment of these unrelated indication, the need for adjustment of doses and duration and the important of making the culture and sensitivity test.

\section{Conclusion}

Drug use evaluation of ceftriaxone with respect to indication manipulates incorrect prescription practice so the specificity of prescribing pattern of ceftriaxone was less; it means empirical therapy was more than specific therapy. Co-administration of ceftriaxone with other drugs was more than it alone, while only a small proportion interact with it, but the most interaction types was serious. The cost is of too high which account for more than 80 Sudanese pounds, and major of patients had insurance.

\section{Financial Resources}

The authors would like to acknowledge that this paper was part of a thesis conducted at the Omdurman Islamic University, Khartoum, Sudan.

\section{References}

1. Helms R, Quan D (2006) Textbook of therapeutics: drug and disease management.

2. Torok E, Moran E, Cooke F (2009) Oxford Handbook of Infectious Diseases and Microbiology. Oxford Medicine Online.
3. Acceptance F, Resistance TO (2000) Post penicillin antibiotics: from acceptance to resistance?. Witn Semin.

4. Brody JE (2004) The Merck Manual of Medical Information. pp: 234.

5. Lee H, Jung D, Yeom JS, Son JS, Jung SI, et al. (2009) Evaluation of ceftriaxone utilization at multicenter study. Korean J Intern Med 24(4): 374-380.

6. Pinto Pereira LM, Phillips M, Ramlal H, Teemul K, Prabhakar P (2004) Third generation cephalosporin use in a tertiary hospital in Port of Spain, Trinidad: need for an antibiotic policy. BMC Infect Dis 4(1): 59.

7. Ibrahim ME, Bilal NE, Hamid ME (2012) Increased multi-drug resistant Escherichia coli from hospitals in Khartoum state, Sudan. Afr Health Sci 12(3): 368-375.

8. Ayinalem G, Gelaw B, Belay A, Linjesa J (2013) Drug use evaluation of ceftriaxone in medical ward of Dessie Referral Hospital, North East Ethiopia. Int J Basic Clin Pharmacol 2(6): 711.

9. Shimels T, Bilal AI, Mulugeta A (2015) Evaluation of Ceftriaxone utilization in internal medicine wards of general hospitals in Addis Ababa, Ethiopia: A comparative retrospective study. J Pharm Policy Pract 8(1): 1-8.

10. Sileshi A, Tenna A, Feyissa M, Shibeshi W (2016) Evaluation of ceftriaxone utilization in medical and emergency wards of Tikur Anbessa specialized hospital: A prospective cross-sectional study. BMC Pharmacol Toxicol 17(1): 1-10.

11. Afriyie DK, Amponsah SK, Dogbey J, Agyekum K, Kesse S, et al. (2017) A pilot study evaluating the prescribing of ceftriaxone in hospitals in Ghana: findings and implications. Hosp Pract (1995) 45(4): 143-149. 